\title{
Graphene reinforced nanocomposites: 3D simulation of damage and fracture
}

\author{
Dai, Gaoming; Mishnaevsky, Leon
}

\section{Published in:}

Computational Materials Science

Link to article, DOI:

10.1016/j.commatsci.2014.08.011

Publication date:

2014

Document Version

Peer reviewed version

Link back to DTU Orbit

Citation $(A P A)$ :

Dai, G., \& Mishnaevsky, L. (2014). Graphene reinforced nanocomposites: 3D simulation of damage and fracture. Computational Materials Science, 95, 684-692. https://doi.org/10.1016/j.commatsci.2014.08.011

\section{General rights}

Copyright and moral rights for the publications made accessible in the public portal are retained by the authors and/or other copyright owners and it is a condition of accessing publications that users recognise and abide by the legal requirements associated with these rights.

- Users may download and print one copy of any publication from the public portal for the purpose of private study or research.

- You may not further distribute the material or use it for any profit-making activity or commercial gain

- You may freely distribute the URL identifying the publication in the public portal

If you believe that this document breaches copyright please contact us providing details, and we will remove access to the work immediately and investigate your claim 


\title{
GRAPHENE REINFORCED NANOCOMPOSITES: 3D SIMULATION OF DAMAGE INITIATION AND EVOLUTION
}

\author{
Gaoming Dai", Leon Mishnaevsky Jr." \\ Department of Wind Energy, Technical University of Denmark, Ris $\varnothing$ Campus, \\ Frederiksborgvej 300 \\ 4000, Roskilde, Denmark
}

\begin{abstract}
:
3D computational model of graphene reinforced polymer composites is developed and applied for the analysis of damage and fracture mechanisms in the composites. The graphene/polymer interface properties are determined using the inverse modeling approach. The effect of composite structure, in particular, of the aspect ratio, shape, clustering, orientation and volume fraction of graphene platelets on the mechanical behavior and damage mechanisms of nanocomposites are studied in computational experiments. It was shown that the Young modulus of the nanocomposite increases with increasing the aspect ratio, volume content, elastic properties of interface layer, and decreasing the degree of intercalation. The tensile strength follows similar tendencies, except for the aspect ratio and clustering degree, where the opposite effects are observed. Nanocomposites with homogenous structures demonstrate much lower Young modulus and strength as compared with aligned, plane graphene sheet reinforcement. It was further concluded that the structural imperfections of graphene reinforcement (like crumpling shape or random misalignment) have considerable effect on the composite performances,
\end{abstract}

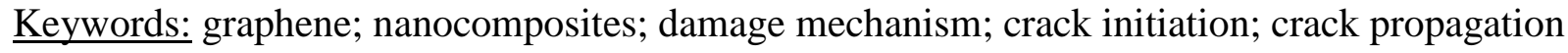

\section{Introduction}

Graphene, a strong and light two-dimensional allotrope of carbon, attracted a growing interest of research community and industry in recent years [1-3]. The materials he material can be used, among others, as reinforcement in polymer composites, thus, potentially ensuring drastic improvement of properties of the composites $[4,5]$.

In order to explore the potential of graphene nanoreinforcements for the polymer strengthening, and to find the recommendations for the computational design of the composites, computational models linking the microstructure of the graphene reinforced composites with their mechanical properties and strength are necessary. A number of computational models of graphene sheets and graphene reinforced composites have been developed in recent years.

Many models are based on atomistic, hybrid and combined atomistic-continuum (FE) and nanoscale continuum approaches [1, 10-[12]. A detailed review of the methods of modeling of graphene and graphene based composites including Quantum Chemistry approach, Molecular

* Corresponding authors: ggda@dtu.dk; lemi@dtu.dk 
Dynamics, Monte Carlo simulation and other techniques is published by Zhang et al. [13]. Quite often, molecular dynamics simulations are employed to analyze the properties of graphene sheets and their interaction with polymer matrix. So, Zhang and Gu [14] used molecular dynamics simulations to determine elastic modulus, fracture stress and fracture strain of graphene. They demonstrated that mechanical properties of graphene are much more sensitive to the temperature changes than to the layer numbers in the multilayer graphene. $\mathrm{Li}$ et al. [15] also used MD simulations to characterize multilayer graphene reinforced epoxy composites. They considered two configurations (graphene layers parallel and perpendicular to polymer/graphene interface). It was shown that the strength of composite similar in both cases, while the damage mechanisms can be different: "the parallel case exhibits cohesive yield with strain localization and nano-void formation within the bulk polymer while the case with graphene sheets oriented normal to the interface exhibit interfacial debonding". Shiu and Tsai [16] used molecular dynamics (MD) simulation, to evaluate the mechanical properties of the graphene reinforced nanocomposites, with different morphologies (graphene platelets, intercalated graphene and intercalated graphene oxide). It was shown that the composites with intercalated graphene have a higher Young's modulus, than do those with graphene platelets. The graphene oxide was shown to be the best reinforcement of the three considered cases. Still, it remains a challenge to link the results of the molecular dynamics simulations to the continuum mechanics and strength models of the composites at the macrolevel, characterizing their service properties. So, more and more works are directed toward the development of coupled MD-continuum mechanical models or purely multiscale mechanical models of graphene composites. So, Motazeri and Rafii-Tabar [11] employed a combined method, based on the molecular dynamics, molecular structural mechanics, and FEM, and determined the elastic constants of nanocomposites. Chandra et al. [17] used a multiscale FEM modeling approach to analyze the mechanical behavior of nanocomposites, and evaluate the effect of the orientation of graphene sheet on the stiffness of the composite. On the basis of the shear lag analysis, Young et al [5] that efficient reinforcement can be realized only if the size of flake is large enough (more than $30 \mu \mathrm{m}$ ). Summarizing these studies, one can see that computational simulations represent a rather efficient tool to determine the damage and deformation mechanisms of the graphene based composites.

While molecular dynamics can deliver rather detailed information on the damage mechanisms, the linkage between the nano structure level and bulk specimen level is not so easy to establish. The continuum mechanics/FEM based approaches can be easily linked to specimen/service properties model, but they should include the correct nanoscale level structure information. Further, the interface properties (which have apparently decisive effect on the properties of graphene reinforced polymers) are not always considered in the above models.

Still, the combination of atomistic and continuum approaches could be considered as the most powerful computational tool to address the problems associated with different scales [18].

In this paper, we seek to develop purely continuum mechanical models of the graphene reinforced nanocomposites, and employ this model to the analysis of microstructure-strength relationships. The objective is to explore factors controlling the strength and damage in graphene reinforced nanocomposites using the methods of micromechanics. In particular, we pay attention to the graphene/polymer interface effects, as well as the role of structural defects (weak interfaces, clustering, various orientations, etc.) on the damage initiation and fracture of the composites. In order to take into account the interface effects, we employ so-called 
"effective interface models", in which the thin layer surrounding the nanoreinforcement is assumed to be a third material, with specific properties, and these properties are determined using the inverse modeling approach. The effects of nanocomposite structure, in particular, effects of the graphene platelet aspect ratio, shape, clustering, orientation and volume fraction, on the mechanical behavior and damage mechanisms of graphene nanocomposite are studied in computational experiments.

\section{Computational model and materials properties}

In this section, we present the computational approach, the method of damage and fracture simulation and the properties of the phases (graphene and epoxy) found in literature.

\subsection{D Finite element model of graphene monolayer composite}

In order to carry our systematic computational studies of the microstructure-strength relationships of graphene reinforced polymers, a number of 3D computational models reflecting the nanocomposite structures should be generated. For this, we employ a a special Python based software code developed in [19-23], and adapt it to the typical structures of graphene based composites (i.e., very high aspect ratio; thin layers). Using the code, a number of 3D FE models of graphene reinforced composites (see examples in Figure 1) were automatically generated. The graphene sheets morphology (orientation, clustering -exfoliated or intercalated, aspect ratio) were taken into account during the model generation process. The reinforcing graphene sheets were randomly distributed in the polymer matrix and (in some models) randomly oriented. To take into account the interface effect, the generalized effective interface layer concept was used [24-26] (see more details below).
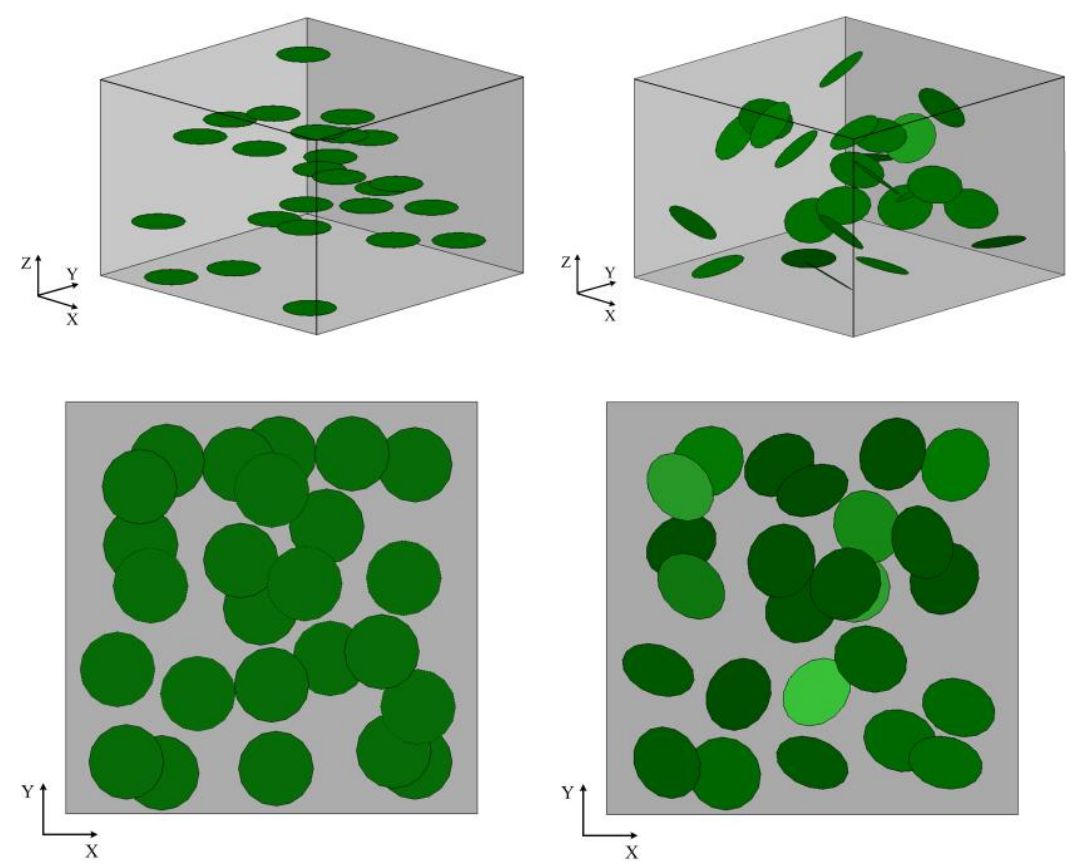

a.

b.

Fig. 1 Examples of 3D unit cell models of graphene reinforced composites

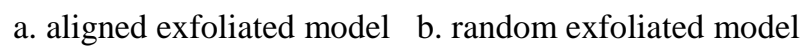

\subsection{Implementation of damage analysis of graphene reinforced composites}


The procedure of numerical simulation of damage evolution in nanocomposites includes two steps: damage onset and damage propagation [19, 32 ].

The initial defects are introduced by subjecting the unit cell to a quasi-static load.The onset of a crack in a graphene reinforced composite is governed by the maximum principal stress criterion, which can be defined as $f=\left\{\left\langle\sigma_{\max }\right\rangle / \sigma_{\max }^{0}\right\}$ [33]. Here, $f$ denotes the maximum principal stress ratio and the damage crack will be formed when $f=1 . \quad{ }_{\max }^{0}$ stands for the maximum allowable principle stress and the symbol \langle\rangle is Macaulay brackets which lets the $\sigma_{\max }$ has the alternative value of 0 or $m_{\mathrm{ix}}$ when $m_{\mathrm{ix}}<0$ or $\sigma_{\max } \geq 0$, respectively.

To model the crack propagation in graphene reinforced composites, 3D power law model is used here [34]. The power law can be described as $\left(G_{I} / G_{I c}\right)^{\alpha}+\left(G_{I I} / G_{I I C}\right)^{\beta}+\left(G_{I I I} / G_{I I I}\right)^{\gamma} \geq 1$. The symbol $G_{\square}$ denotes the strain energy release rate parameters and indices $I, I I, I I I$ stand for the three fracture modes. The index $c$ represents the critical values of strain energy release rate (the fracture toughness). $\alpha, \beta, \gamma$ are parameters and are assigned the value of 1 .

The numerical simulation works are carried out with the commercial FE code ABAQUS/STANDARD (version 6.11). Different 3D models of graphene reinforced composites are subjected to uniaxial tensile loading (displacement) $u$ along the Zaxis direction. The three-dimensional 4-node linear tetrahedron element C3D4 is used for meshing. The virtual crack closure technique (VCCT) [35, 36] is used to calculate the strain energy release rate and the linear elastic fracture mechanics (LEFM) approach and the framework of extended-FEM (xFEM) method [37-40] are involved to implement the crack evolution analysis. The comparison of $x F E M$, cohesive zone approach and the damageable layers model as applied for thew modelling cracking in composites has been carried out and presented in [32, 41]. Comparing the methods, the authors demonstrated that these approaches give very similar results. With view of the efficiency of XFEM, this method is used in the current simulations.

\subsection{Material properties}

On the basis of an analysis of literature data, the following mechanical and strength properties of graphene and matrix could be identified. :

Graphene and graphene/polymer interface: thickness of the graphene sheet $0.335 \mathrm{~nm}$; aspect ratios of $3.5 \ldots .7 \times 10^{4}$ [5]; typical diameter of 10-40 $\mu \mathrm{m}$ [42]; Young's modulus and shear modulus of $1050 \mathrm{GPa}$ [5-7] and $311 \mathrm{GPa}$ [53]; tensile strength of $130 \mathrm{GPa}$ [7]. maximum shear stress of $2.3 \mathrm{MPa}$ at the edge of the sheet; graphene-matrix interfacial shear stress is on the order of $0.25-0.8 \mathrm{MPa}$ and with a maximum value of $1 \mathrm{MPa}$ [42]; fracture stress of a graphene sheet is larger than $100 \mathrm{GPa}$ and the failure strain loading is on the order of $20 \%$ [28].

Epoxy matrix: Young's modulus of $2.13 \mathrm{GPa}$ [50]; shear modulus of $1 \mathrm{GPa}$ [42]; tensile strength of $49.9 \mathrm{MPa}$ [50]; threshold/critical strain energy release rate of matrix [19,23], namely, $G_{I \mathrm{th}}=0.06 \mathrm{~kJ} / \mathrm{m}^{2}, \mathrm{G}_{I \mathrm{C}}=0.173 \mathrm{~kJ} / \mathrm{m}^{2}, \mathrm{G}_{I I \mathrm{th}}=0.24 \mathrm{~kJ} / \mathrm{m}^{2}, \mathrm{G}_{I I \mathrm{C}}=0.648 \mathrm{~kJ} / \mathrm{m}^{2}$, $\mathrm{G}_{I I I \mathrm{th}}=0.306 \mathrm{~kJ} / \mathrm{m}^{2}, \mathrm{G}_{I I I \mathrm{C}}=0.850 \mathrm{~kJ} / \mathrm{m}^{2}$ for mode $I, I I$ and $I I I$, respectively. 


\section{Properties of graphene/polymer interface: Inverse modeling}

The graphene/ matrix interfaces have a significant influence on the mechanical properties and strength of nanocomposites [3, 28].

In this section, the interface properties between the graphene sheets ad polymer matrix are investigated using the inverse modeling. As noted in [26], the extraordinary effect of nanoreinforcement on the mechanical properties of nanocomposites (i.e., when let's say, addition of a very fraction of nanoreinforcement leads to a drastic increase of the elastic properties, far beyond the expected "rule of mixture" estimations applicable for microreinforcements) is connected with the interaction between nanoreinforcements (with very high surface area) and polymer matrix, leading to the formation of the polymer layer with modified, perturbed chain structure. This layer of perturbed polymer surrounding the nanosheets is the main source of the extraordinary material strengthening, and is modeled here via the "effective interface layer".

In [24, 29], the computational model of nanocomposite was developed, which includes (apart from usual matrix and reinforcement phases) a third phase, corresponding to the polymer volume surrounding the nanoreinforcements (here, graphene flakes) and demonstrating different properties than the rest of matrix, due to the perturbed polymer chain structures near the nanoparticles. This third phase is presented as "interphase layer" and the model is called "effective interface model".

Here, we seek to estimate the properties of the "effective interface" around the graphene sheet, employing the inverse modeling approach [27]. Novoselov, Young and their colleagues at the University Manchester [42-44] studied the change in the strain distribution on graphene, and observed that the strain across the flake is uniform at the applied strain up to $0.6 \%$. After that cracks form in polymer coating layers, with the graphene remaining intact. As a result, the interfacial shear stress in the fragments is reduced to about $0.25 \mathrm{MPa}$. Here, the cases experimentally studied in [43] are simulated numerically. The model contains three material phases: matrix, interface and graphene monolayer. The graphene monolayer has a thickness $0.34 \mathrm{~nm}$, aspect ratio 2000, and is assumed to be surrounded by effective interface with a thickness of $1 \mathrm{~nm}$ (estimated as one half of the minimum separation between monolayer sheets given as $2 \mathrm{~nm}$, see [5]). The model dimensions are $18.4 \mu \mathrm{m} \times 10.4 \mu \mathrm{m} \times 0.3634 \mu \mathrm{m}$. The effective interface layers were modeled automatically using the developed code.

\subsection{Static analysis: Determination of elastic properties of the interface layer}

Here, we use the iterative approximation method to determine the Young modulus of the effective interface layer which is supposedly surround graphene sheets in the matrix.

First, we evaluate overall range of possible variation of the effective interface. Apparently, the effective interface cannot be stiffer than the graphene sheet. Since "relatively poor adhesion between the graphene and polymer layers" was observed [28] and following Wang et al. [26], (who took the Young's modulus of the interface as $1 / 14$ of matrix stiffness, $4.2 / 14=0.3 \mathrm{GPa}$ ), we take the low limit at the level of $0.15 \mathrm{GPa}$.

Models shown in Figure 2 are subject to applied strains $0.4 \%$ and $0.6 \%$ along the X-direction. The assumed elastic module of interface is $3.695 \mathrm{GPa}$ (close to that of epoxy).

Figure 3 gives four strain distributions along the central line/X coordinate of the graphene sheet 
for different interface stiffnesses. Under the $0.4 \%$ strain loading, the strain is remains at the almost constant level (except for a tiny drop at the middle of the graphene sheet) through the graphene sheet but fall down to 0 at both sides. This corresponds well to the results from [28]. Under the strain loading of $0.6 \%$, the strain level at the central part of graphene sheet is reduced as the stiffness of the effective interface increases. It reaches the level of $0.4 \%$ when the interface has a stiffness of $3.74 \mathrm{GPa}$. The perfect "M" shape of the strain distribution along the length of graphene sheet corresponds to the experimental results by Young et al., [28]. for the Young modulus of the interface $3.74 \mathrm{GPa}$.

Thus, the Young modulus of the interface layer $3.74 \mathrm{GPa}$ can be used in the following simulations.

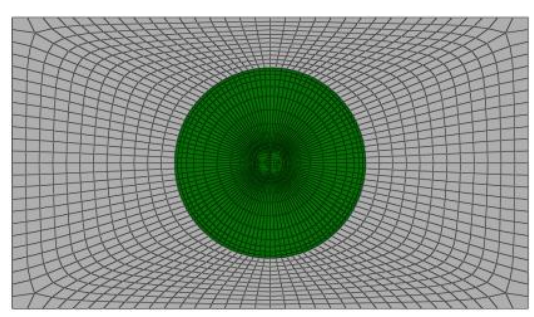

a.

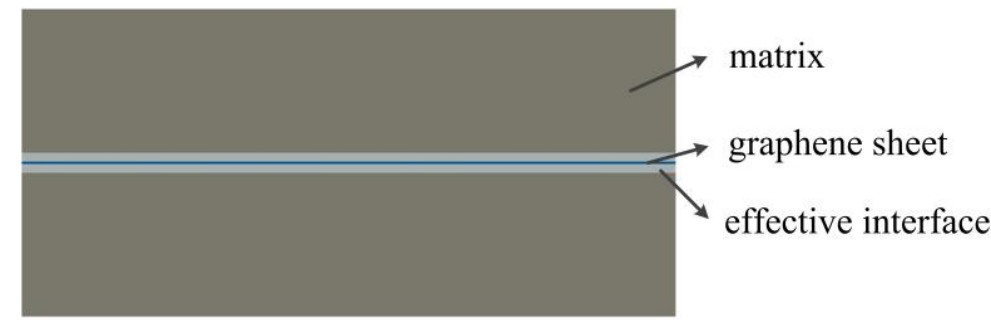

b.

Fig. 2 A model to the inverse determination of the effective interface properties

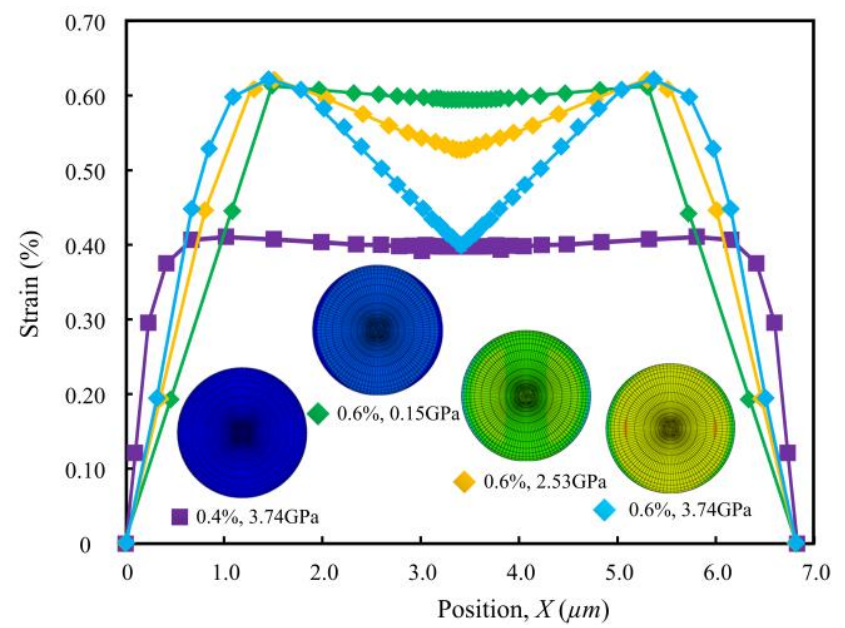

Fig. 3 Strain distribution along the $\mathrm{X}$-coordinate on the central line of graphene

\subsection{Damage analysis: inverse estimation of the strength of graphene/polymer interface}

Here, we seek to estimate the strength of the interface layer, using the inverse modeling. Again, we start with rough estimation of the strength variation range. In [19], the nanoclaypolymer interface layer has a tensile strength of $82 \mathrm{MPa}$. Given the "relatively poor adhesion between the graphene and polymer layers" [28], and higher aspect ratio of graphene, we take the range the tensile strength of graphene sheets to be between $82 \mathrm{MPa}$ and $8.2 \mathrm{MPa}$ (10 times smaller). 
Again, the model shown in Figure 2 is subject to $0.8 \%$ strain loading along the $\mathrm{X}$-direction (as in the experiments in [28]). The correct interface layer strength should correspond to the situation when cracks are initiated in interface when the strain loading level reaches $0.6 \%$ [28]. The Young modulus obtained in the section 3.1 is assigned to the interface layer.

Fig. 4 gives the tensile stress-strain curves for the different interface strengths. Fig. 4a shows that cracks are formed under strain $0.4 \%$ in the model with the interface strength $18.25 \mathrm{MPa}$. At the applied strains $0.6 \%$ and $0.8 \%$, the material is already highly damaged. In the model with interface strength of $74.5 \mathrm{MPa}$ (Figure $4 \mathrm{~b}$ ), there is no damage even under $0.8 \%$ strain loading. In the model with the interface strength of $32.4 \mathrm{MPa}$ (Fig 4c), the material is intact under $0.4 \%$ strain. The crack is initiated under $0.6 \%$ strain loading, and the material gets quickly cracked under $0.8 \%$ strain. Thus, the approximately strength of the effective interface can be taken (approximately) $32.4 \mathrm{MPa}$.

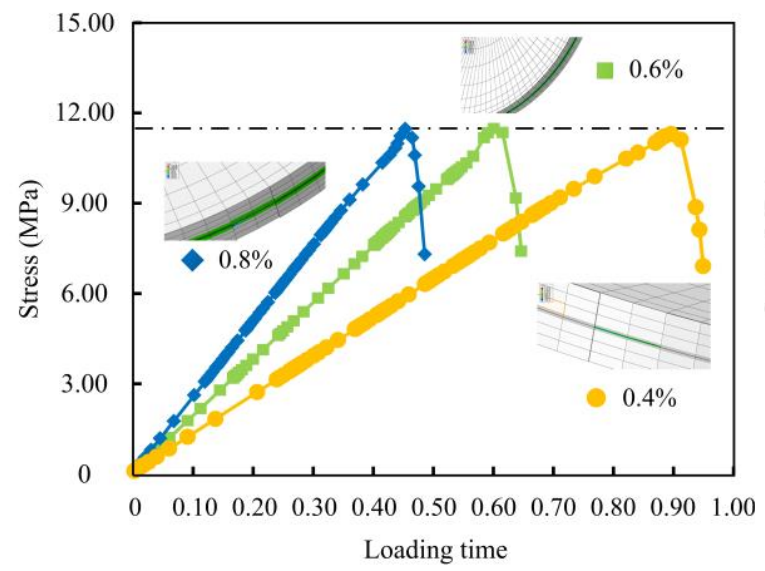

A

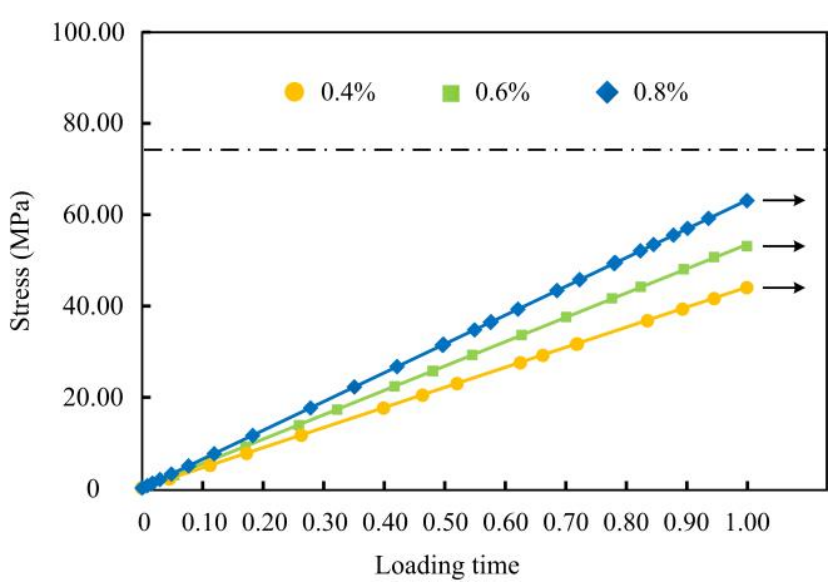

b

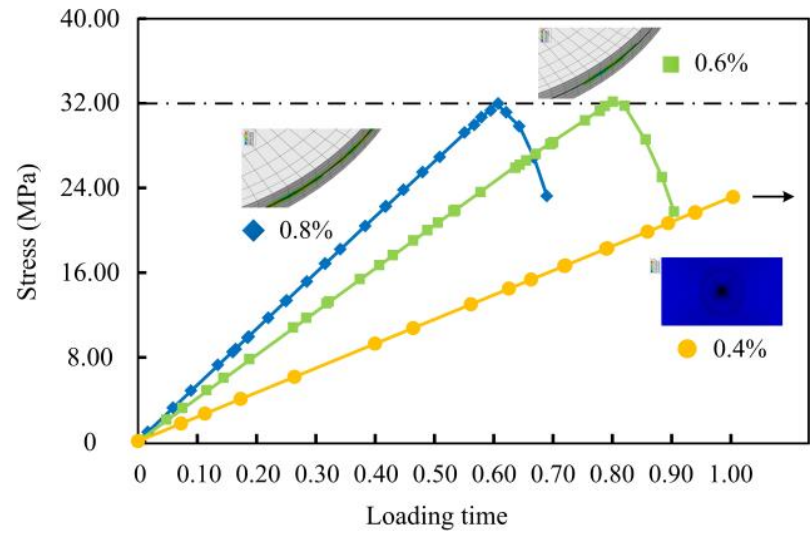

(c)

Fig. 4 Stress-loading time relationship under different strain loading and different interface strength as a. 18.2 MPa, b. $74.5 \mathrm{MPa}$, c. $32.4 \mathrm{MPa}$

Further, we studied the horizontal (parallel to X-direction) shear stress distribution along the central line of a graphene sheet under different applied strains and different interface strengths (see Fig. 5). It is found that the shear stress on the edge of graphene sheet increases (from 3.2 $\mathrm{MPa}$ to $4.8 \mathrm{MPa}$ ) as the strain loading increases (from $0.4 \%$ to $0.6 \%$ ) before the interface was damaged. There is a sharp decrease of the shear stress level (from 4.8 MPa to 0.32 MPa) after the model was damaged (under $0.8 \%$ strain loading). These results correspond well to the 
observations by Young et al. [28].

Thus, in this section, we used inverse modeling and the experimental data from Young et al. [28] to evaluate the elastic and strength properties of the graphene/epoxy interface (considered as a thin layer). The obtained values: Young modulus 3.74 GPa and strength $32.4 \mathrm{MPa}-$ will be used in our further simulations. One should be noted that these results obtained by fitting numerical results (and not the exact solutions) to the experimental results. Thus, the precision of such fitting results is limited.

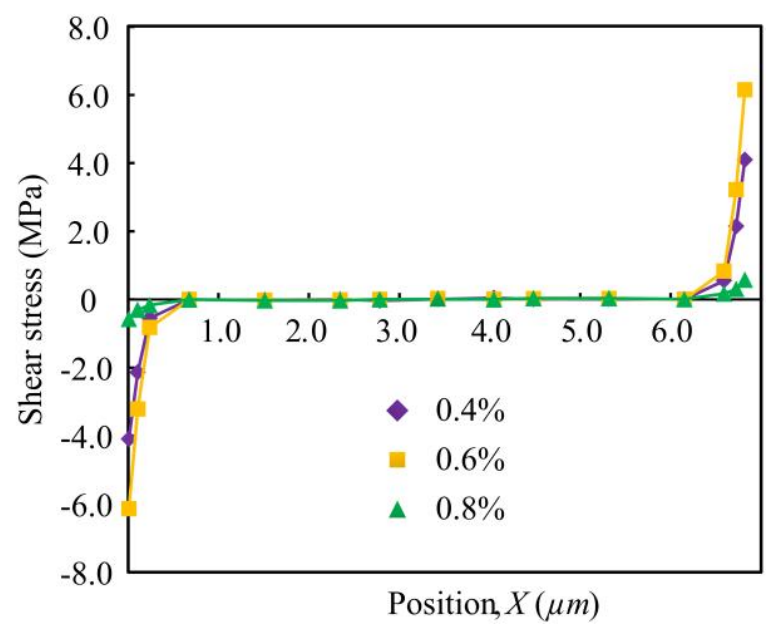

Fig. 5 Shear stress level along the $X$ direction

\section{Graphene morphology and crunching effect}

Mechanical properties of graphene reinforced composites strongly depend on the content and distribution of graphene sheets [3]. The graphene sheets orientation (aligned and random), aspect ratio, clustering degree (intercalated and exfoliated) and volume fraction (graphene loading in the composites) have effects on the mechanical behavior of composites. In this section, we seek to analyze the effect of these parameters on the mechanical behavior of the nanocomposites.

\subsection{Effect of graphene sheet orientation on the deformation and strength of nanocomposites}

In this section, we consider the effect of graphene orientation on the mechanical properties of the composites. Several 3D unit cell models with 25 graphene sheets with aspect ratio 2000 (graphene fraction of $0.50 \%$ ) and varied graphene orientations (aligned/random) were generated (Fig. 1). The graphene nanosheets in the aligned models are parallel to the X-Y plane while the graphene nanosheets in the random model are assigned random angles with the plane (in the range of $0-65^{\circ}$ ).

Fig. 6 the stress-strain curves for aligned and random arranged graphene models subject to normal and axial loadings. (Here, $\mathrm{Z}$ direction loading, means the loading normal to the graphene sheets, and $\mathrm{X}$ direction means the loading along the graphene sheets).

The composites with aligned graphene sheets demonstrate a higher Young's modulus (3.519 $\mathrm{GPa}$ in $\mathrm{X}$ direction and $3.270 \mathrm{GPa}$ in $\mathrm{Z}$ direction versus $1.915 \mathrm{GPa}$ in $\mathrm{X}$ direction and 1.839 $\mathrm{GPa}$ in the $\mathrm{Z}$ direction for the material with random orientations) and higher ultimate strength 
(106.764 $\mathrm{MPa}$ and $89.56 \mathrm{MPa}$ in $\mathrm{X}$ and $\mathrm{Z}$ direction, respectively, when versus $40.9117 \mathrm{MPa}$ and $38.82 \mathrm{MPa}$ for the random models). Thus, the misalignment of nanosheets leads to the reduction of Young's modulus of the composites by $43.76 \%$, and to the $56.65 \%$ decrease of strength (with the $\mathrm{Z}$ direction loading).

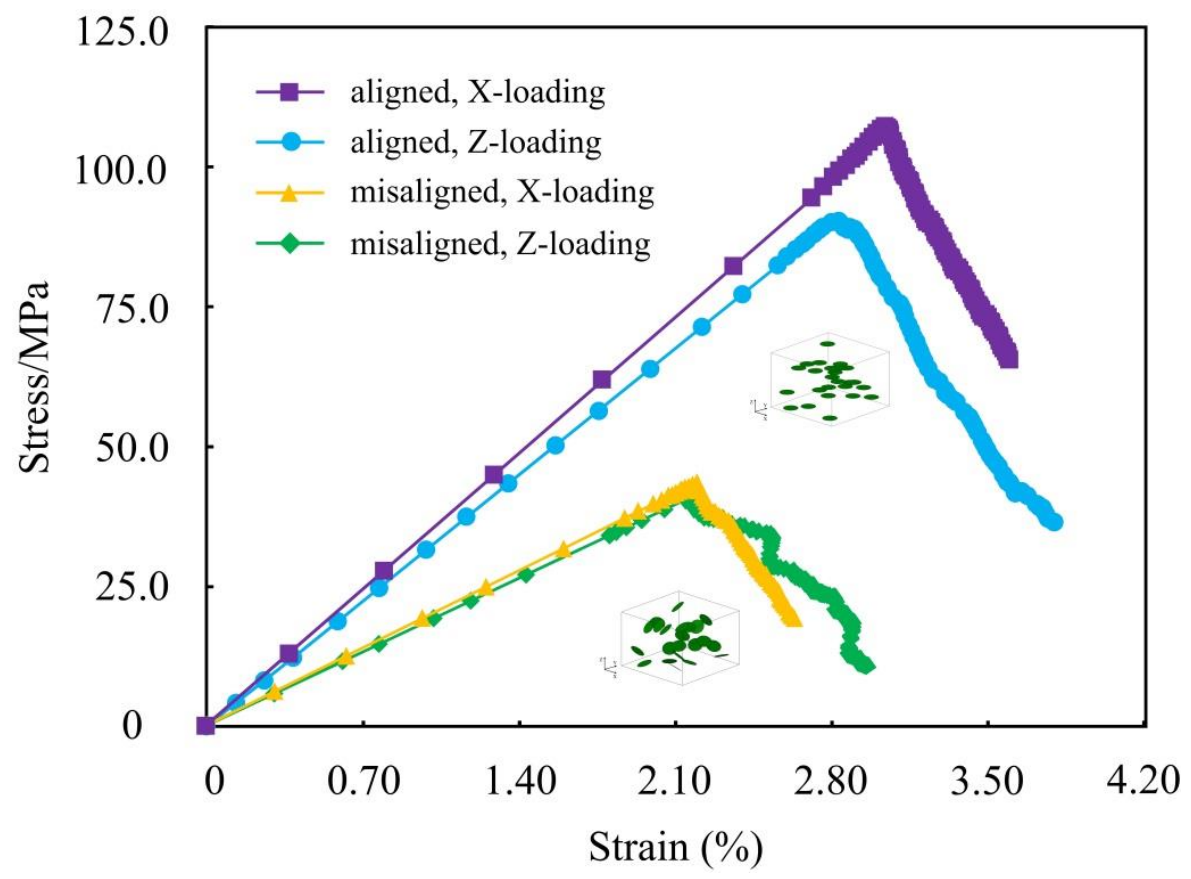

Fig. 6 Strain-stress relationship composites with aligned and random graphene reinforcement

The stress-strain curve of the model with random reinforcement is more zigzagged than that with aligned reinforcement. This can mean that the misalignment of graphene sheets can delay the crack propagation.

Fig. 7 gives the cracks morphology in aligned and random model, respectively. One can see that a crack initiates at the graphene-matrix interface in both cases. Then it grows throughout the whole interface region and propagates into the matrix. Cracks meet in the matrix and merge together to form the main crack. One can observe that the main damage mechanisms in the case of a composite with aligned sheets are debonding and crack deflection.

Further damage mechanisms such as crack pinning and crack bridging are observed in the case with randomly oriented sheets.
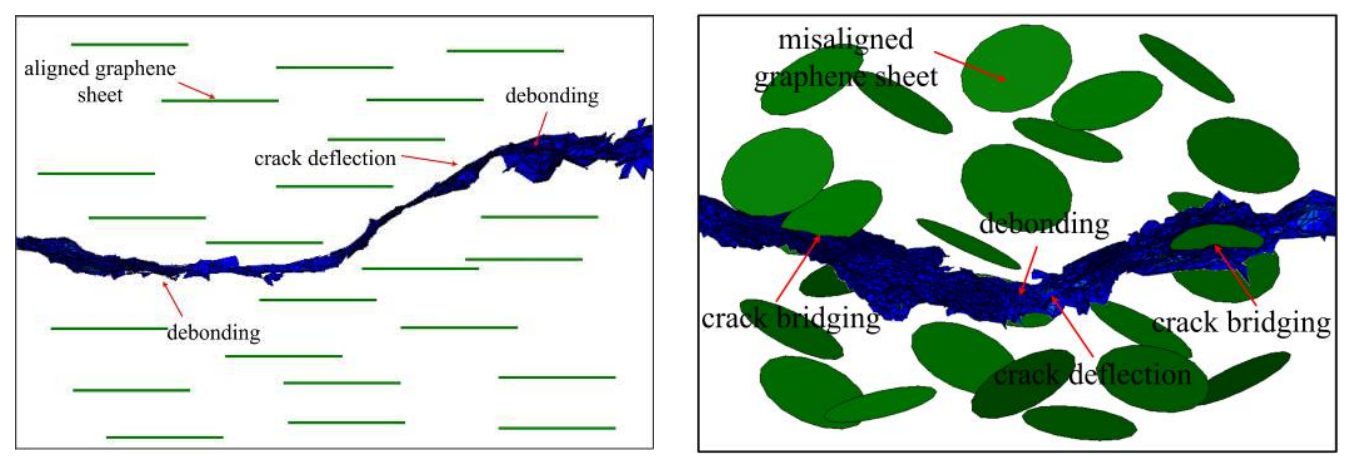

a.

Fig. 7 Crack morphology in an aligned and random model 


\subsection{Effect of aspect ratio of graphene on the deformation and strength of nanocomposites}

Here, we consider the effect of the aspect ratio of graphene sheets on the mechanical behavior $f$ graphene reinforced polymer composites.

Several 3D unit cells with varied aspect ratios of graphene sheets were generated. The volume content of graphene was $0.5 \%$ (25 graphene sheets per unit cell). The aspect ratios of graphene sheets were taken 1000, 2000, 10000 and 20000.

Figure 8 shows the stress-strain curves for the considered cases. One can see that the material with the aspect ratio of graphene 1000 model shows the highest tensile strength (114.71 MPa) and lowest Young's modulus $(2.911 \mathrm{GPa})$, while the nanocomposite with aspect ratio sheets 20000 show the lowest tensile strength (46.94 MPa, 59\% lower) but largest Young's modulus (3.667 GPa, 26\% higher). Similar results have been reported by Boo et al. [45].

It is known that for the fibrous reinforcement, there exist a critical aspect ratio after which the positive effect of enlargement of fiber length becomes weak or even negligible. In different works, the critical aspect ratios for carbon nanotubes are estimated at the level of 200..300 [46, 47]. As shown by Mortazavi et al. [48], the critical aspect ratio increases when the contrast in properties of the matrix and reinforcement increases. In our simulations, the considered aspect ratios are significantly higher than the values estimated in these and other works. That explains relatively weak influence of the aspect ratios of graphene reinforcements observed in the simulations.

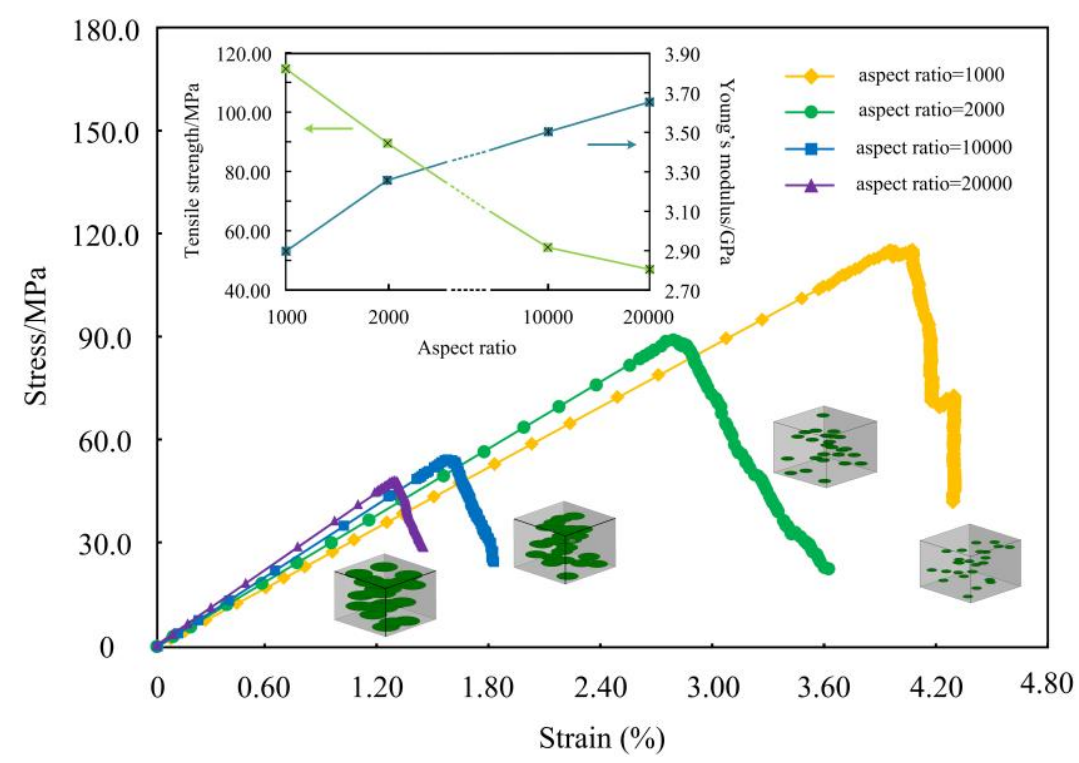

Fig. 8 Graphene reinforced composites: aspect ratio effects

\subsection{Effect of graphene clustering}

Currently, the goal to fabricate truly exfoliated graphene composite is still far from being achieved [3]. Graphene sheets in the composites are quite often stacked or intercalated [4].

In this section, the effect of clustering of graphene sheets on the mechanical properties of nanocomposites is studied. 
Several 3D unit cell models with 25 exfoliated graphene sheets and with 5 clusters (each cluster with 5 graphene sheets) as well as with 3 graphene monolayers plus 4 clusters with clustering degree of 2 ( 2 graphene sheets in one cluster), 3 clusters with clustering degree of 3 and one 5 degree cluster were generated and tested in the simulations. The volume content of graphene was $0.5 \%$.

As differed from the modeling concept of nanocomposites used by Dai and Mishnaevsky [22] earlier, the effective interfaces in the current models were built only around graphene sheets or cluster (i.e., between graphene sheets or graphene clusters, and the matrix), but not between graphene sheets inside the clusters. This modification was motivated by the experimental observations by Gong et al. [30], who estimated the thickness of graphene trilayer as $1 \mathrm{~nm}$ (just 3 times of the monolayer thickness, $0.34 \mathrm{~nm}$ ) and Mahmoud [31] (who measured the thickness of four layers graphene sheets as $1.4 \mathrm{~nm}, 4$ times of a single graphene sheet). It also means that the internal debonding between graphene in each graphene multilayer cluster was neglected.

Figure 9 shows the stress-strain curves for the unit cells with different degrees of clustering.

One can see that the tensile strength increases as the clustering degree of nanoreinforcement increases (from $89.56 \mathrm{MPa}$ for the exfoliated structure model to $108.55 \mathrm{MPa}$ for the model with cluster degree of 5, what gives an increase of 21.2\%). The Young's modulus of nanocomposite decreases with the increasing clustering (from 3.270 GPa to $2.454 \mathrm{GPa}$, with the decrease of 25\%). When the graphene sheets are clustered, their effective aspect ratio decreases. On the other side, mechanical properties of the clusters become transversely isotropic and not homogenous. This has a strong influence on the mechanical properties of composites.

\subsection{Volume content effects}

Now, we seek to analyze the effect of the volume fraction of graphene in the graphene reinforced composites. A number of unit cell models with different graphene contents $(0.25 \%$, $0.50 \%$ and $1.00 \%$ ) were generated and tested.

Fig.10a shows that the volume fraction of the graphene in the nanocomposites has a significant effect on the damage mechanical behavior of graphene reinforced nanocomposites. The unit cell model with graphene content $1.00 \%$ has the highest tensile strength $(111.67 \mathrm{MPa})$ and Young's modulus $(5.906 \mathrm{GPa})$ while the model with $0.25 \%$ graphene loading has the lowest tensile strength and Young's modulus of $75.16 \mathrm{MPa}$ and $2.407 \mathrm{GPa}$, respectively. It is apparent that the mechanical performance of the composites exhibits a dramatic improvement due to the reinforcement of graphene sheets.

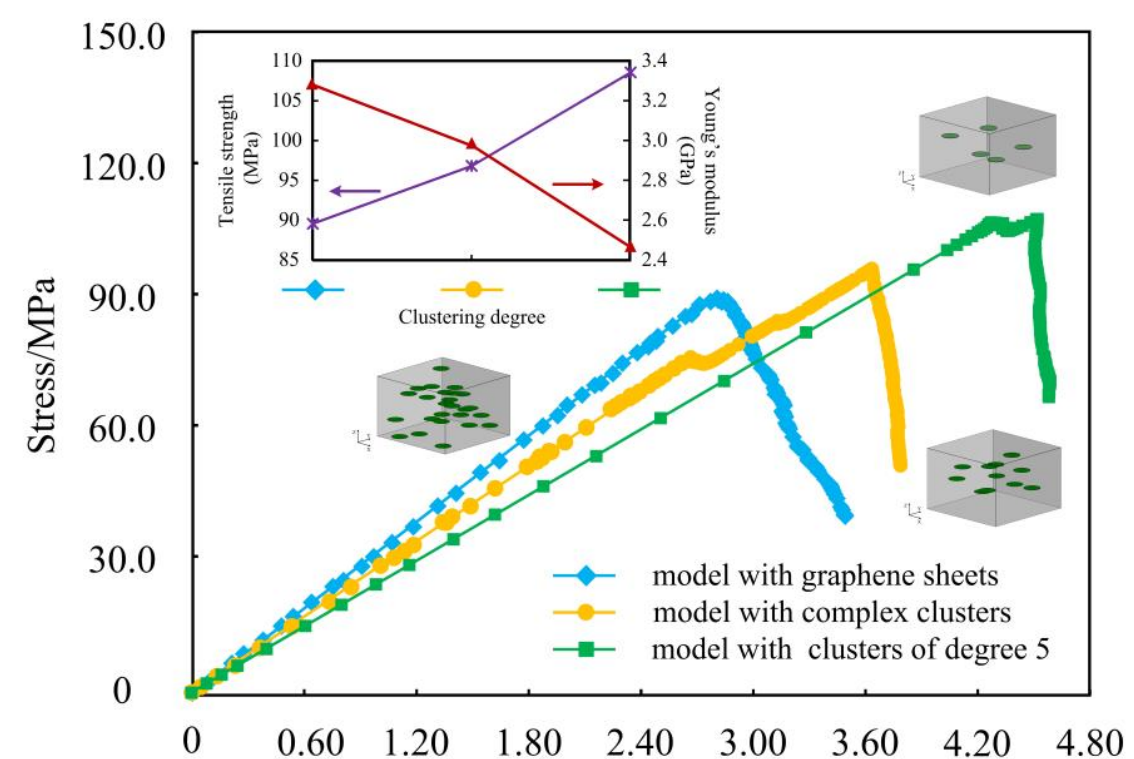


Fig. 9 Clustering effect upon the mechanical behavior of graphene reinforced composites (a) and models with different clustering degrees

If comparing the graphene reinforced nanocomposite $(0.50 \%$ graphene $)$ with pure polymer, one can see that the tensile strength and the Young's modulus is $79.44 \%$ higher (from 49.9 $\mathrm{MPa}$ to $89.56 \mathrm{MPa}$ ) and $53.52 \%$ higher (from $2.13 \mathrm{GPa}$ to $3.27 \mathrm{GPa}$ ), in the nanocomposite. Thus, the effect of graphene nanoreinforcements is far stronger than the "rule of mixture" estimation.

It can be concluded that the graphene reinforcement has a very strong effect on the mechanical properties of composites. The tensile strength and Young's modulus of the composites both increased as the volume fraction of graphene sheets increased.

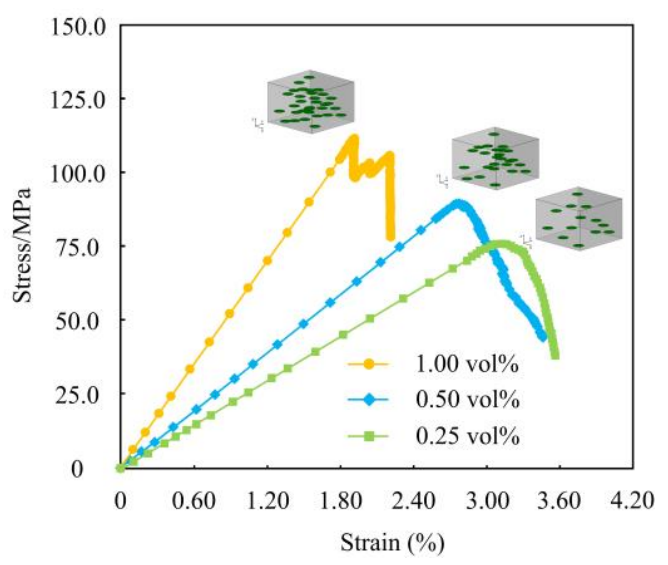

Fig. 10 Volume fraction effects on the mechanical behavior of graphene reinforced nanocomposites a: stress-strain curves b: tensile strength and Young's modulus versus graphene loading

\section{Graphene shape and interfacial properties effect}

According to Singh et al [3], "controlling the folding, crumpling and bending of graphene materials" and "understanding of the interfacial structure and properties" are two of the five main challenges in producing idle graphene reinforced composites.

In this section, the effects of graphene sheet crumbling and the interfacial properties on the nanocomposite performances are studied numerically.

\subsection{Effect of crumpled graphene on the deformation and strength of nanocomposites}

Graphene sheets in polymers are not always ideal discs, but have rather often bent, crumpled and waived shapes [4]. When dispersed in the polymer matrix, graphene sheets can adopt wavy or wrinkled forms, which may also reduce the moduli. 
In this section, we study the effect of crumpled graphene sheet on the mechanical properties of graphene reinforced composites.

The polygon graphene sheet (which Gong et al. [42] considered in their work) is modeled here (Fig. 11b-d). The polygon graphene sheet has a dimension of $12 \mu \mathrm{m}$ and $34 \mu \mathrm{m}$ in $\mathrm{X}$ and Y direction, respectively. The whole model has a dimension of $24 \mu m \times 45 \mu m \times 15 \mu m$ along the $X$, $Y$ and $Z$ direction. Also, a crumpled version of the polygon graphene sheet model was generated on the basis of the polygon model (Fig. 11c).

Fig. 11a shows the stress-strain curves, estimated elastic and strength properties for these two cases. As expected, the flat graphene model is strongly preferable over the crumpled graphene. The flat graphene model shows a tensile strength of $83.529 \mathrm{MPa}$ while the crumpled one is $38.824 \mathrm{MPa}$, (53.52\% decrease). Also, Young modulus is $9.49 \%$ lower for the crumpled graphene (from $2.107 \mathrm{GPa}$ to $1.907 \mathrm{GPa}$ ).

We can see that shape imperfections of graphene sheets lead to much stronger reduction of strength, than elastic properties of composite.

One should be also noted that graphene is in fact a highly flexible material while in our models it is represented as a highly rigid material (due to its high elastic modulae). While it is beyond the current study, this aspect of graphene defdormation will be incorporated in the following simulations which are under way now. This issue is especially important for crumpled graphene.
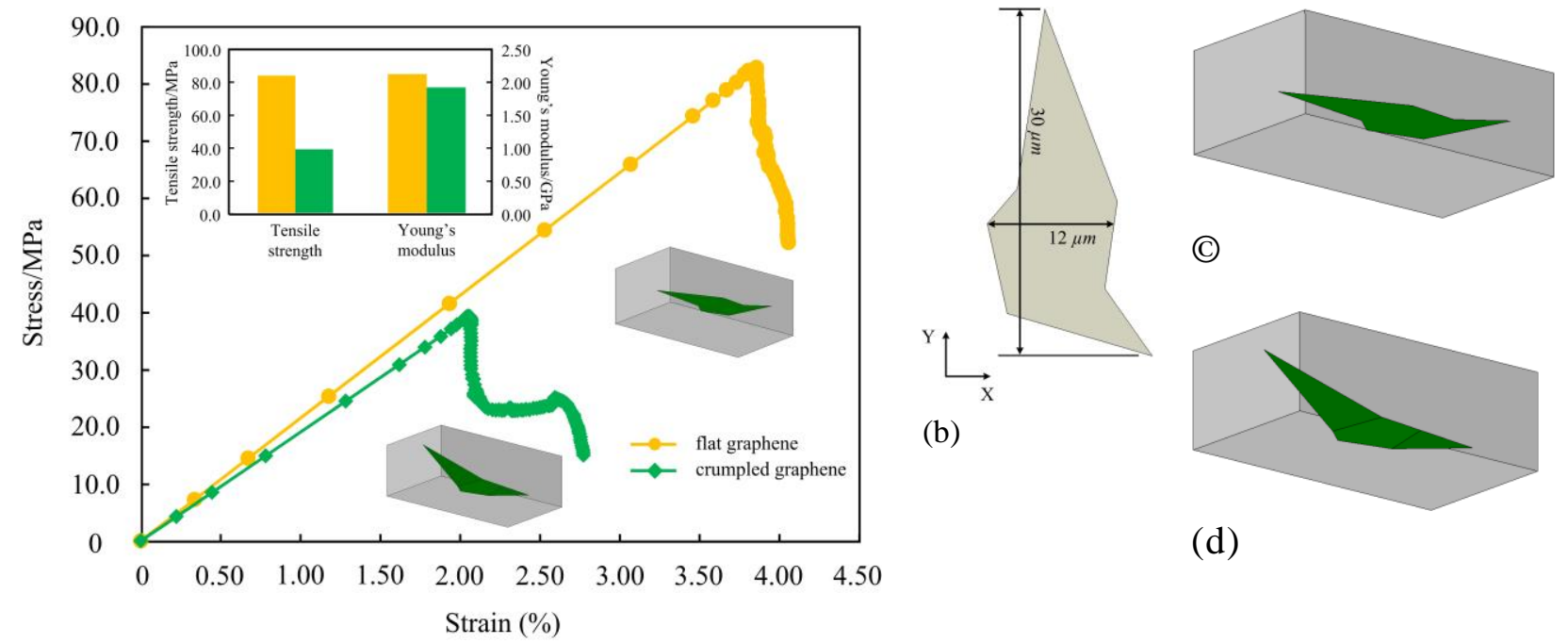

(C)

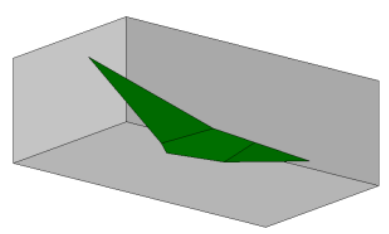

(d)

(a)

Fig. 141Crumpled effects upon the graphene reinforced composites(a), and schema of crumpled (d) and plain (c) sheets and their dimensions (b)

\subsection{Interfacial strength and its effect on the material behavior}

In several works, the potential of chemical functionalization of graphene for improving the interfacial bonding characteristics between graphene and polymers, and ultimately, its reinforcing properties, were studied $[4,51,52]$.

Let us consider here the effect of the interface layer strength on the mechanical properties and strength of the composite. 
A number of 3D unit cell models with 25 aligned graphene sheets (aspect ratio 2000, and volume content $0.5 \%$ ) were generated. Different interfacial strengths were assigned to the interface layers $(67,198$ and $514 \mathrm{MPa})$.

Figure 12 shows the results of simulations. One can see that the material with strong interfaces (514 MPa) ensures the highest tensile strength (127.94 MPa), Young's modulus (3.687 GPa) and largest elongation till failure. The material with weakest interface (67 MPa) has the lowest tensile strength $(50.29 \mathrm{MPa})$, Young's modulus $(2.873 \mathrm{GPa})$ and elongation. Thus, the stronger interface is indeed an important resource to improve the mechanical properties of graphene reinforced composites. Young et al [28] also noticed that that forming strong interfaces between matrix and graphene can provide the nanocomposites with "reasonable stiffness, and strength".

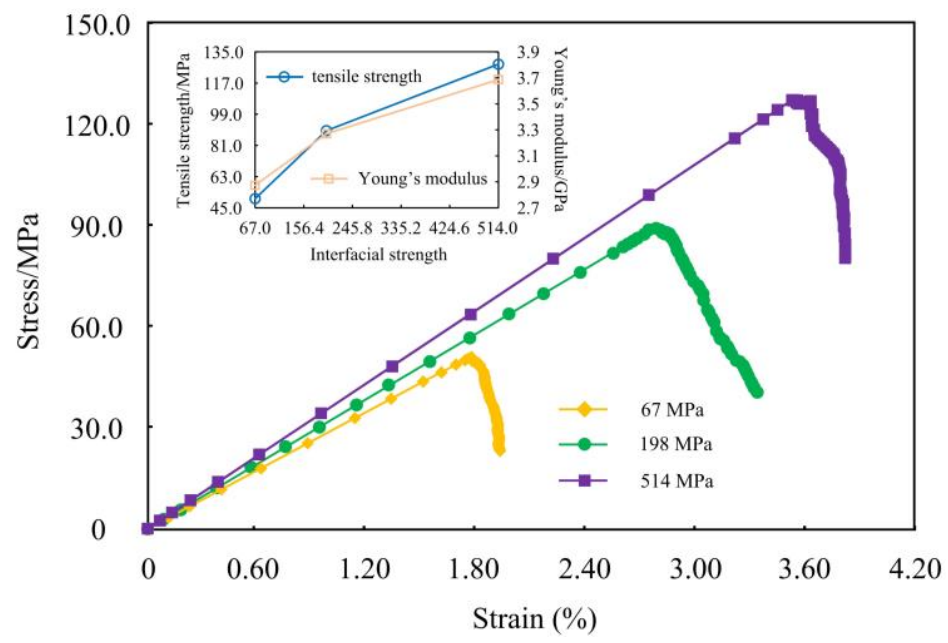

Fig. 12 Interfacial properties effects on the material behavior

\section{Discussion}

It is of interest to compare the results of numerical experiments with some literature data. Using the inverse analysis, we determined the elastic modulus of the polymer layer surrounding the graphene sheets, which is $3.74 / 2.14=75 \%$ higher than $t$ he elastic modulus of polymer matrix. This corresponds to the results by Shiu and Tsai [16], who observed the morphology of polymer chains near the graphene sheets and found that " the local density close to the graphene is relatively high, and there are greater amounts of high density polymers in the intercalated graphene nanocomposites. These high amounts of high density polymer are responsible for the enhanced thermal and mechanical properties in the intercalated nanocomposites".

In the section 4.4, we observed that if comparing the graphene reinforced nanocomposite $(0.50 \%$ graphene) with pure polymer, the tensile strength and the Young's modulus are $79.44 \%$ and $53.52 \%$ higher, respectively. According to Liang et al. [50], the tensile strength and Young's modulus increase by $76 \%$ and $62 \%$, respectively when $0.41 \mathrm{vol} \%$ of graphene sheets are introduced into PVA matrix. Zhao et al. [52], that the tensile strength of a graphene sheets reinforced composite with $0.6 \%$ graphene is $73 \%$ higher than that of pure matrix, while the Young's increases by $150 \%$ (however, the aspect ratio of graphene sheets in their materials is larger and is up to 3000-6000). These evaluations are in fact very close to our computational estimations of the effect of graphene reinforcement on the polymer properties $(79 \%, 53 \%)$.

In the section 4.1, we observed the cracks morphology in the composite with aligned and 
randomly oriented sheets.. It was observed that a crack initiates at the graphene-matrix interface in both cases. This corresponds to the observation by Gong et al. [8] that the adhesion between graphene and polymer is relatively poor. Then crack grows throughout the whole interface region and propagates into the matrix, they deflect on the nanosheets. Also, Shadlou et al. [9] observed the crack deviations on nanoparticles and crack propagation along the interfaces as most typical crack growth mechanisms in epoxy nanocomposites with various carbon nanoreinforcements.

In section 4.3, it was demonstrated that the graphene clustering leads to the higher stiffness of the graphene reinforced composites. This is confirmed by the results of the molecular dynamics simulations by Shiu and Tsai [16] who showed that composites with intercalated graphene have a higher Young's modulus, than those with graphene platelets. The effect of the graphene aspect ratio on the composite properties, observed in section 4.2 is relatively weak: only $26 \%$ higher stiffness as a result of the 20 times higher aspect ratio. However, this corresponds also to results by Chandra et al. [17], who observed that “... the stress-strain curves obtained do not show a considerable dependence of the results on the length of the graphene reinforcement".

\section{Conclusions}

In this paper. microstructure-mechanical properties and -strength relationships for graphene reinforced polymer nanocomposites are investigated numerically, using $3 \mathrm{D}$ micromechanical unit cell models. The elastic and strength properties of the thin interface layer between graphene sheets and epoxy polymer are determined using the inverse modeling method. The effect of microstructure of graphene based composite, in particular, aspect ratio, shape (crumpled versus flat) and orientation of graphene sheets, their clustering, on the stress-strain curves, tensile strength and elastic properties of the nanocomposite are studied.

Summarizing the presented numerical experiments, one can draw the following conclusions. The Young modulus of the nanocomposite increases with increasing the aspect ratio, volume content, strength of interface layer and decreases with the higher degree of graphene sheet clustering,. The tensile strength follows similar tendencies, except for the aspect ratio and clustering degree, where the opposite effects are observed. Randomization of nanoplatelet orientation leads to the strong reduction of Young modulus and strength of the nanocomposite.

The factors influencing the elastic properties and strength of nanocomposites can be ranked as follows (from stronger to weaker effects): crumpled graphene shape (only for strength: 50\% reduction of strength only due to the shape crumpling, but only $10 \%$ reduced stiffness) $\rightarrow$ graphene sheet misalignment $(\sim 50 \%$ reduction of stiffness and strength because of misalignment) $\rightarrow$ volume content of graphene (50\% higher stiffness and 2.5 times higher strength at 4 times higher content of graphene) -> interface strength ( 7 times higher interface strength gives only 2.5 times higher composite) and clustering degree (only $25 \%$ difference between highly clustered and exfoliated) $->$ the aspect ratio of sheets ( $60 \%$ lower strength and $26 \%$ higher stiffness due to 20 times higher aspect ratio). (The ranking is conditional, still, it shows that the structural imperfections in graphene reinforced composites, like sheet crumpling and misalignment, have the strongest effect on the composite properties).

\section{Acknowledgement:}

The author gratefully acknowledges the financial support of the Danish Council for Strategic Research (DSF) via the Sino-Danish collaborative project "High reliability of large wind 
turbines via computational micromechanics based enhancement of materials performances" (Ref. no. 10-094539). Furthermore, the author is grateful to the DSF for its support via the Danish Centre for Composite Structures and Materials for Wind Turbines (DCCSM) (Contract no. 09-067212).

\section{References:}

1. A.K. Geim, K.S. Novoselov. The rise of graphene. Nat. Mater. 2007; 6: 183-190.

2. A.K. Geim. Graphene: status and prospects. Science 2009; 324: 1530-1534.

3. V. Singh, D. Joung, L. Zhai, S. Das, S.I. Khondaker, S. Seal. Graphene based materials: Past, present and future. Progress in Materials Science 2011; 56: 1178-1271.

4. J.R. Potts, D.R. Dreyer, C.W. Bielawski, R.S. Ruoff. Graphene-based polymer nanocomposites. Polymer 2011; 52: 5-25.

5. R.J. Young, I.A. Kinloch, L. Gong, K.S. Novoselov. The mechanics of graphene nanocomposites: A review. Composites Science and Technology 2012; 72: 1459-1476.

6. C. Lee, X.D. Wei, J.W. Kysar, J. Hone. Measurement of the elastic properties and intrinsic strength of monolayer grapheme. Science 2008; 321: 385-388.

7. F. Liu, P.B. Ming, J. Li. Ab initio calculation of ideal strength and phonon instability of graphene under tension. Phys. Rev. B 2007; 76: 064120.

8. L. Gong, R.J. Young, I.A. Kinloch, I. Riaz, R. Jalil, K.S. Novoselov. Optimizing the reinforcement of polymer-based nanocomposites by graphene. ACS Nano 2012; 6: 2086-2095.

9. S. Shadlou, E. Alishahi, M.R. Ayatollahi. Fracture behavior of epoxy nanocomposites reinforced with different carbon nano-reinforcements. Composite Structures 2013; 95: 577-581.

10. Y. Chandra, F. Scarpa, R. Chowdhury, S. Adhikari, J. Sienz. Multiscale hybrid atomistic-FE approach for the nonlinear tensile behavior of graphene nanocomposites. Composites Part A: Applied Science and Manufacturing 2013; 46: 147-153.

11. A. Montazeri, H. Rafii-Tabar. Multiscale modeling of graphene- and nanotube-based reinforced polymer nanocomposites. Physics Letters A 2011; 375: 4034-4040.

12. M.M. Shokrieh, R. Rafiee. Prediction of Young's modulus of graphene sheets and carbon nanotubes using nanoscale continuum mechanics approach. Materials \& Design 2010; 31: 790-795.

13. T. Zhang, Q. Xue, S.Zhang, M.Dong Theoretical approaches to graphene and graphene-based materials, Nano Today, Volume 7, Issue 3, 2012, Pages 180-200

14. Y.Y. Zhang, Y.T. Gu, Mechanical properties of graphene: Effects of layer number, temperature and isotope. Computational Materials Science, Volume 71, 2013, Pages 197-200

15. F. Li, N. Yan, Y. Zhan, et al.Probing the reinforcing mechanism of graphene and graphene oxide in natural rubber, Journal of Applied Polymer Science Volume: 129 Issue: 4 Pages: 2342-2351 2013

16. S.C. Shiu, J.L. Tsai, Characterizing thermal and mechanical properties of graphene/epoxy nanocomposites , Composites Part B: Engineering, Volume 56, January 2014, Pages 691-697

17. Y. Chandra, F. Scarpa, R. Chowdhury, S. Adhikari, J. Sienz, Multiscale hybrid atomistic-FE approach for the nonlinear tensile behaviour of graphene nanocomposites, Composites Part A: Applied Science and Manufacturing, Volume 46, March 2013, Pages 147-153

18. B. Mortazavi, O. Benzerara, H.Meyer, J. Bardon, S. Ahzi, Combined molecular dynamics-finite element multiscale modeling of thermal conduction in graphene epoxy nanocomposites Carbon 60, 356-365, 2013

19. G.M. Dai, L. Mishnaevsky Jr., Fatigue of multiscale composites with secondary nanoplatelet reinforcement: 3D computational analysis, Composites Science and Technology, Vol. 91, 2014, pp. 71-81

20. L. Mishnaevsky Jr., G.M. Dai, Hybrid carbon/glass fiber composites: Micromechanical analysis of structure-damage resistance relationship, Computational Materials Science, Vol. 81, 2014, pp. 630-640

21. L. Mishnaevsky Jr., G.Dai, Hybrid and hierarchical polymer composites: Computational modelling of structure-properties relationships, Composite Structures, DOI:10.1016/j.compstruct.2014.06.027

22. G.M. Dai, L. Mishnaevsky Jr., Damage evolution in nanoclay-reinforced polymers: a three-dimensional computational study, Composites Science \& Technology, 74 (2013) 67-77

23. G.M. Dai, L. Mishnaevsky Jr., Fatigue of hybrid carbon/glass composites: 3D Computational modelling Composites Science \& Technology, Vol. 94, 2014, pp. 71-79 
24. Odegard G.M., Clancy T.C., Gates T.S.. Modeling of the mechanical properties of nanoparticle/polymer composites. Polymer 2005; 46: 553-62.

25. R.D. Peng, H.W. Zhou, H.W. Wang, L. Mishnaevsky Jr. Modeling of nano-reinforced polymer composites: Microstructure effect on the Young's modulus, Computational Materials Science, 60 (2012) 19-31

26. Wang HW, Zhou HW, Peng RD, Mishnaevsky Jr L. Nanoreinforced polymer composites: 3D FEM modeling with effective interface concept. Compos Sci Technol 2011; 71: 980-8.

27. L.Mishnaevsky Jr, Micromechanical analysis of nanocomposites using 3D voxel based material model, Composites Science \& Technology, 72 (2012) 1167-1177

28. R.J. Young, L. Gong, I.A. Kinloch, I. Riaz, R. Jalil, K.S. Novoselov. Strain mapping in a graphene monolayer nanocomposite. ACS Nano 2011; 5: 3079-3084.

29. Mishnaevsky Jr L, Brondsted P. Three-dimensional numerical modelling of damage initiation in UD fiber-reinforced composites with ductile matrix. Mater Sci Eng: A 2008; 498: 81-6.

30. L. Gong, R.J. Young, I.A. Kinloch, I. Riaz, R. Jalil, K.S. Novoselov. Optimizing the reinforcement of polymer-based nanocomposites by graphene. ACS Nano 2012; 6: 2086-2095.

31. W.E. Mahmoud. Morphology and physical properties of poly (ethylene oxide) loaded graphene nanocomposites prepared by two different techniques. European Polymer Journal 2011; 47: 1534-40.

32. Wang HW, Zhou HW, Mishnaevsky Jr L, Brondsted P, Wang LN. Single fibre and multifibre unit cell analysis of strength and cracking of unidirectional composites. Computational Materials Science 2009; 46: 810-20.

33. Greco F, Leonetti L, Blasi PN. Non-linear macroscopic response of fiber-reinforced composite materials due to initiation and propagation of interface cracks. Eng Fract Mech 2012; 80: 92-113.

34. Goyal VK, Johnson ER, Davilia CG. Irreversible constitutive law for modeling the delamination process using interfacial surface discontinuities. Compos Struct 2004; 65: 289-305.

35. Rybicki EF, Kanninen MF. A finite element calculation of stress intensity factors by a modified crack closure integral. Eng Fract Mech 1977; 9: 931-8.

36. Krueger R. Virtual crack closure technique: history, approach and applications. Appl Mech Rev 2004; 57: 109-43.

37. Belytschko T, Gracie R, Ventura G. A review of extended/generalized finite element methods for material modeling. Modell Simul Mater Sci Eng 2009; 17: 1-24.

38. Belytschko T, Black T. Elastic crack growth in finite elements with minimal remeshing. Int J Numer Meth Eng 1999; 45: 601-20.

39. Stolarska M, Chopp DL, Moes N, Belyschko T. Modelling crack growth by level sets in the extended finite element method. Int J Numer Meth Eng 2001; 51: 943-60.

40. Sukumar N, Moes N, Moran B, Belytschko T. Extended finite element method for three-dimensional crack modeling. Int J Numer Meth Eng 2000; 48: 1549-70.

41. H.W. Wang, H.W. Zhou, H.W. Ji, X.C. ZhangApplication of extended finite element method in damage progress simulation of fiber reinforced composites, Materials \& Design, Volume 55, March 2014, Pages 191-196

42. L. Gong, I.A. Kinloch, R.J. Young, I. Riaz, R. Jalil, K.S. Novoselov. Interfacial stress transfer in a graphene monolayer nanocomposite, Adv. Mater. 2010; 22: 2694-2697.

43. R.J. Young, L. Gong, I.A. Kinloch, I. Riaz, R. Jalil, K.S. Novoselov. Strain mapping in a graphene monolayer nanocomposite. ACS Nano 2011; 5: 3079-3084.

44. L. Gong, R.J. Young, I.A. Kinloch, I. Riaz, R. Jalil, K.S. Novoselov. Optimizing the reinforcement of polymer-based nanocomposites by grapheme. ACS Nano 2012; 6: 2086-2095.

45. Boo W.J., Sun L., Warren G.L., Moghbelli E., Pham H., Clearfield A., et al. Effect of nanoplatelets aspect ratio on mechanical properties of epoxy nanocomposites. Polymer 2007; 48: 1075-82.

46. A. Needleman, et al.,Effect of an interphase region on debonding of a CNT reinforced polymer composite, Composites Science and Technology, Volume 70, Issue 15, 15 December 2010, Pages 2207-2215

47. J.N. Coleman et al. ,Small but strong: A review of the mechanical properties of carbon nanotube-polymer composites, Carbon, Volume 44, Issue 9, August 2006, Pages 1624-1652

48. B. Mortazavi, J. Bardon, S.Ahzi Interphase effect on the elastic and thermal conductivity response of polymer nanocomposite materials: 3D finite element study, Computational Materials Science, Volume 69, March 2013, Pages 100-106 
49. J.Y Wang, Z.Q. Li, G.L. Fan, H.H. Pan, Z.X. Chen, D. Zhang. Reinforcement with graphene nanosheets in aluminum matrix composites. Scripta Materialia 2012; 66: 594-597.

50. J.J. Liang, Y. Huang, L. Zhang, Y. Wang, Y.F. Ma, T.Y. Guo, Y.S. Chen. Molecular-level dispersion of graphene into poly (vinyl alcohol) and effective reinforcement of their nanocomposites. Adv. Funct. Mater. 2009; 19: 1-6.

51. C. Lv, Q.Z. Xue, D. Xia, M. Ma. Effect of chemisorption structure on the interfacial bonding characteristics of graphene-polymer composites. Applied Surface Science 2012; 258: 2077-2082.

52. X. Zhao, Q.H. Zhang, D.J. Chen. Enhanced mechanical properties of graphene-based poly (vinyl alcohol) composites. Macromolecules 2010; 43: 2357-2363.

53. J.L. Tsai, S.H. Tzeng, Y.T. Tzou. Characterizing the fracture parameters of a graphene sheet using atomistic simulation and continuum mechanics. International Journal of Solids and Structures 2010; 47: 503-509. 